\title{
Bone marrow involvement in pediatric malignancies: a comparison study of Positron emission tomography- computed tomography and bone marrow biopsy
}

\author{
Özlem Tezol ${ }^{1 \oplus}$, Fatih Sağcan ${ }^{1 \oplus}$, Pınar Pelin Özcan ${ }^{2 \oplus}$, Elvan Çağlar Çıtak ${ }^{3 \oplus}$ \\ Departments of ${ }^{1}$ Pediatrics, ${ }^{2}$ Nuclear Medicine and ${ }^{3}$ Pediatric Oncology, Mersin University Faculty of Medicine, Mersin, Turkey.
}

\begin{abstract}
Background and objectives. The comparison of Positron emission tomography- computed tomography (PET$\mathrm{CT}$ ) and bone marrow biopsy (BMB) modalities in detecting bone marrow disease is an up to date research topic. In this study, we aimed to compare the results of PET-CT and BMB procedures in detecting bone marrow involvement in pediatric malignancies.
\end{abstract}

Method. At the time of diagnosis, PET-CT imaging and BMB performed patients' data were evaluated, retrospectively. Malign diagnoses were Hodgkin's lymphoma in 23 (30.7\%), non-Hodgkin's lymphoma in 20 (26.7\%), neuroblastoma in 11 (14.7\%), Ewing sarcoma in 10 (13.7\%), Langerhans cell histiocytosis in $6(8 \%)$, and rhabdomyosarcoma in $5(6.6 \%)$ patients.

Results. Bone marrow involvement was detected in 39 (52\%) of 75 patients. Bone marrow involvement was identified by both PET-CT and BMB in $18(46.1 \%)$ patients, by only PET-CT in $12(30.7 \%)$ patients, by only BMB in $9(23 \%)$ patients. The sensitivity of PET-CT was $66 \%$, specificity was $75 \%$, positive predictive value was $60 \%$, and negative predictive value was $80 \%$. Sensitivity, spesificity, positive and negative predictive values of PETCT were different in before mentioned malignancy groups.

Conclusion. PET-CT may not have high sensitivity and specificity to identify bone marrow involvement for each type of cancer. The approach of using bone marrow biopsy and PET-CT as complementary modalities seems reliable.

Key words: bone marrow involvement, pediatric malignancies, PET-CT.

Cancer a major cause of mortality, is rare in childhood. Bone marrow involvement (BMI) is a sign of advanced disease and poor prognosis in childhood malignancies. In the pediatric population, at the time of diagnosis, Hodgkin's lymphoma (HL) has BMI in $4-14 \%$ of patients, and stage-IV non-Hodgkin's lymphoma (NHL) frequency is $8.4-25.4 \%$ in recent studies. ${ }^{1-3}$ The frequency of BMI in newly-diagnosed pediatric patients with Ewing sarcoma was reported to be $8.5 \%$, with neuroblastoma it was reported to be $54.5 \%{ }^{4,5}$ Bone marrow biopsy (BMB) is a

$凶$ Özlem Tezol

ozlemtezol@hotmail.com

Received 21st January 2019, revised 15th June 2019, 27th July 2019, accepted 29th July 2019. procedure for diagnosis, staging, identification of prognostic risk factors, treatment monitoring in pediatric malignancies. BMB is accepted as a "gold standart" modality in identifying bone marrow involvement.

Positron emission tomography-computed tomography (PET-CT) is the most frequently used imaging method in staging of malign diseases in adult patients. It provides information about the anatomic structures and metabolic activities of tumors, and visualizes the whole body including bone marrow. BMB is an invasive and painful procedure with disadvantages such as general anesthesia need in young children or sedation need in older children, false negative results due to sampling failure, failure in detection of focal involvement. ${ }^{6}$ 
By means of high fluor-18 fluorodeoxyglucose (18F-FDG) avidity of the bone marrow, imaging of the entire marrow, and detecting focal involvements are known as the superiorities of PET-CT. ${ }^{7}$ In case of incompatibility between different imaging procedures, PET-CT imaging has the highest diagnostic accuracy in $90 \%$ of cases, it changes the stage of disease in $61 \%$ of cases, and it changes the clinical approch in $24 \%$ of patients. $^{8}$ There is emerging evidence to suggest that FDG PET-CT has an established role in staging pediatric malignancies, and $\mathrm{BMB}$ can be safely excluded in patients with normal bone marrow on PET-CT. ${ }^{9}$ For instance, Zapata et al. ${ }^{6}$ reported no false negative BMI results with PET-CT in pediatric solid tumors, and Hassan et al. ${ }^{7}$ reported a high sensitivity, specificity and negative predictive value for BMI on PET-CT in pediatric HL, likewise Chen et al. ${ }^{15}$ reported a sensitivity and specificity greater than $90 \%$ in pediatric NHL.

The comparison of PET-CT and BMB modalities in detecting bone marrow disease is a research topic that remains update. In this study, we aimed to compare the results of PET-CT and $\mathrm{BMB}$ modalities in detecting BMI in pediatric malignancies that are known to metastasize to the bone marrow, and to investigate the sensitivity and specificity of PET-CT.

\section{Material and Methods}

Patients' data diagnosed with Hodgkin's lymphoma, non-Hodgkin's lymphoma, Ewing sarcoma, neuroblastoma, rhabdomyosarcoma and langerhans cell histiocytosis (LCH) between June 2008 and June 2018 were evaluated, retrospectively. At the time of diagnosis and before the initiation of systemic therapy, PETCT imaging and BMB performed patients' data were included. Demographic and clinical characteristics were collected as data.

Following 4-6 hours fasting, PET-CT imagings of patients were performed who were hidrated with dextrose free fluid. One hour after 18F-FDG injection the entire body was scaned in 3D mode (Siemens Medical Solusions, Erlangen, Germany and GE Discovery PETCT 610 , US). Imaging results were evaluated by a nuclear medicine specialist who did not know the results of BMBs. PET-CT results of patients with FDG uptake similar to adjacent soft tissues in bone marrow were accepted as negative. Results with FDG uptake equal with primary tumor or more than adjacent tissues in bone marrow were accepted as positive. Diffuse or multifocal FDG uptake patterns were noted.

Bone marrow biopsies were performed by the same pediatric oncologist from the standard iliac crest region before the PETCT scan. Bilateral sampling from iliac crests were performed. All biopsy samples were adequate, and examined histopathologically and immunohistochemically in pathology laboratory. The pathologist performed CD1a/ S100 staining in LCH and choromogranin, synaptophysin, neuron spesific enolase, CD99, desmin, vimentin stainings in small-blueround-cell tumors. Leukocyte common antigen, CD20, CD79a, CD3, CD5, CD15, bcl2, bcl-6, CD30, CD10, CD30, terminal deoxynucleotidyl transferase stainings were performed in lymphomas.

PET-CT result was accepted as true negative if there was not metastasis in bone marrow biopsy, PET-CT result was accepted as true positive if there was metastasis in bone marrow biopsy. Sensitivity, specificity, positive predictive value (PPV), and negative predictive value (NPV) of the PET-CT modality were calculated. The study protocol was approved by the local ethics committee (MEU 2018/315).

\section{Results}

Over a period of ten years, we evaluated 75 patients in whom both BMB and PET-CT procedures were performed. Malign diagnoses were Hodgkin's lymphoma (HL) in 23 (30.7\%), non-Hodgkin's lymphoma (NHL) in 20 (26.7\%), neuroblastoma in 11 (14.7\%), Ewing sarcoma in $10(13.7 \%)$, langerhans cell histiocytosis (LCH) 
in $6(8 \%)$, and rhabdomyosarcoma in $5(6.6 \%)$ patients. Mean (SD) age was 13.0 (4.1) years in HL, 10.6 (4.4) years in NHL, 2.3 (1.6) years in neuroblastoma, 10.5 (4.2) years in Ewing sarcoma, 6.9 (4.8) years in LCH, 9.0 (3.8) years in rhabdomyosarcoma group. Percentage of male patients was $52.1 \%$ in HL, 65\% in NHL, $72.7 \%$ in neuroblastoma, 60\% in Ewing sarcoma, 50\% in $\mathrm{LCH}, 60 \%$ in rhabdomyosarcoma group. BMI was detected in 39 (52\%) patients. The malignancy group with the highest frequency of bone marrow involvement was non-Hodgkin's lymphoma (16 of 20 patients, $80 \%$ ). Diagnoses and BMI frequencies are shown in Table I.

BMI was identified by both PET-CT and BMB in $18(46.1 \%)$ patients, by only PET-CT in $12(30.7 \%)$ patients, by only BMB in $9(23 \%)$ patients. The examples for these groups of patients were given in Figures 1, 2 and 3. For the whole group, the sensitivity of PET-CT was $66 \%$, specificity was $75 \%$, positive predictive value was $60 \%$ and negative predictive value was $80 \%$ (Table II). The sensitivity values of PET-CT for HL, NHL, neuroblastoma, Ewing sarcoma, rhabdomyosarcoma, LCH groups were 100, 50, $75,50,100,100 \%$, respectively. The specificity

Table I. Diagnoses and bone marrow infiltration frequencies.

\begin{tabular}{lc}
\hline Diagnosis & BM infiltration \\
\hline Hodgkin's lymphoma & $9(39.1 \%)$ \\
Non-Hodgkin's lymphoma & $16(80.0 \%)$ \\
Neuroblastoma & $4(36.3 \%)$ \\
Ewing sarcoma & $5(50.0 \%)$ \\
Langerhans cell histiocytosis & $3(50.0 \%)$ \\
Rhabdomyosarcoma & $2(40.0 \%)$ \\
\hline
\end{tabular}

BM: bone marrow.

Table II. Comparison of BMB results \& PET-CT results.

\begin{tabular}{lcc}
\hline \multirow{2}{*}{ PET-CT results } & \multicolumn{2}{c}{ BMB results } \\
\cline { 2 - 3 } & BMB positive & BMB negative \\
\hline Positive & 18 & 12 \\
Negative & 9 & 36 \\
\hline
\end{tabular}

BMB: bone marrow biopsy, PET-CT: positron emission tomography-computed tomography. values of PET-CT for HL, NHL, neuroblastoma, Ewing sarcoma, rhabdomyosarcoma, $\mathrm{LCH}$ groups were $73,50,100,83,100,60 \%$, respectively. The positive predictive values of PET-CT for HL, NHL, neuroblastoma, Ewing sarcoma, rhabdomyosarcoma, LCH groups were $44,60,100,66.6,100,33.3 \%$, respectively. The negative predictive values of PET-CT for HL, NHL, neuroblastoma, Ewing sarcoma, rhabdomyosarcoma, LCH groups were 100, 40, $87.5,71.4,100,100 \%$, respectively.

PET-CT had false negative results in 9 patients. Six of these PET negative BMB positive patients were diagnosed with $\mathrm{NHL}$, and there were generalised hypermetabolic lympadenopathies on their PET-CT scans (SUVmax range $=4.52-$ 28.59). Two of these PET negative BMB positive patients were diagnosed with Ewing sarcoma, and there were tumoral uptakes on their PETCT scans (SUVmax values $=6.87$ and 4.79 ). One of these PET negative and BMB positive patients was diagnosed with neuroblastoma, and there was tumoral uptake on his PET-CT scan $($ SUVmax $=3.73)$.

PET-CT detected abnormal up-take in different bone marrow regions defined as multifocal uptake pattern in $12 \mathrm{BMB}$ negative patients. Increased multifocal F-FDG uptake in 11 patients was in several bone marrow regions except iliac crests (cranium, rib, sternum, humerus, tibia, femur, sacrum). One patient diagnosed with HL had abnormal F-FDG uptake in left iliac crest and negative $\mathrm{BMB}$ result.

Clinical course of BMB negative patients was good as anticipated, these patients had higher one-year survival rates. The distribution of procedures identifying bone marrow involvement according to the diagnosis and one-year survival rates are shown in Table III.

\section{Discussion}

Early diagnosis and disease screening have great importance for pediatric oncology. For the determination of the treatment protocol, $\mathrm{BMI}$ is assessed by BMB, as the gold standard method. 


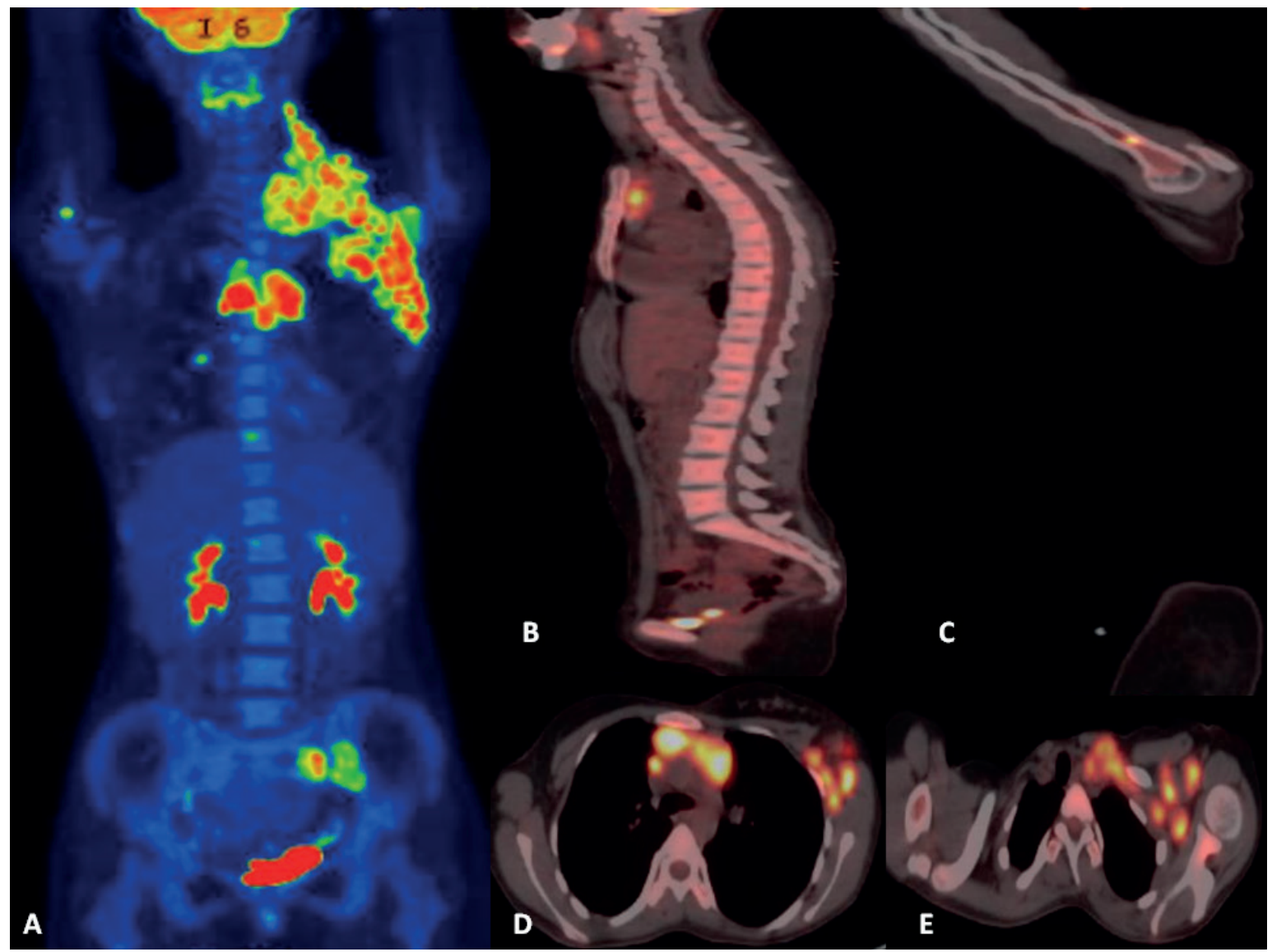

Fig. 1. FDG PET-CT images of a 15 year old female patient with Hodgkin Lymphoma diagnosis. Maximum intensity projection (MIP) image (A), sagittal $(\mathrm{B}, \mathrm{C})$ and axial $(\mathrm{D}, \mathrm{E})$ fusion images demonstrate multipl hypermetabolic lymphadenopaties and bone-bone marrow diffuse and multifocal FDG uptake compatible with lymphoma diagnosis. Bone marrow biopsy was negative for this patient.

PET-CT has found a wide and reliable area of use in terms of diagnosis, accurate staging, and evaluation of treatment response in pediatric malignancies. In pediatric tumor staging, the sensitivity values of PET and PET-CT for all cancers were reported to be $90-97 \%$ and 99 $100 \%$, respectively. ${ }^{10-12}$ In the current study, PETCT showed $66 \%$ sensitivity and $75 \%$ specificity for demonstrating BMI.

Most pediatric studies investigated BMI by PET$\mathrm{CT}$ in patients with lymphoma. PET-CT shows all the lesions detected by $\mathrm{BMB}$, and it is able to differentiate diffuse uniform involvement due to anemia or reactive changes and multifocal bone involvement due to lymphoma. ${ }^{8}$ In a study conducted with 54 patients with a lymphoma diagnosis (31 HL, $24 \mathrm{NHL}$ ), the specificity and positive predictive values were reported to be $100 \%$ for both PET-CT and BMB, and the sensitivity and negative predictive values were $92 \%$ and $54 \%$, respectively for PET-CT, and $98 \%$ and $87 \%$, respectively for $\mathrm{BMB} .{ }^{13}$ Our study showed lower results in whole lymphoma group for PET-CT in detecting BMI compared with BMB (62\% versus 52\% sensitivity, $66 \%$ versus $75 \%$ specificity).

In a study of 784 pediatric patients diagnosed with Hodgkin's lymphoma, $13.3 \%$ of patients had BMI. The sensitivity, specificity, positive and negative predictive values of PET-CT were found to be $93.6,94,53$, and $99.4 \%$, respectively, and this method was reported to be more sensitive than $\mathrm{BMB}$ for detecting BMI. The authors recommended that BMB should only be 

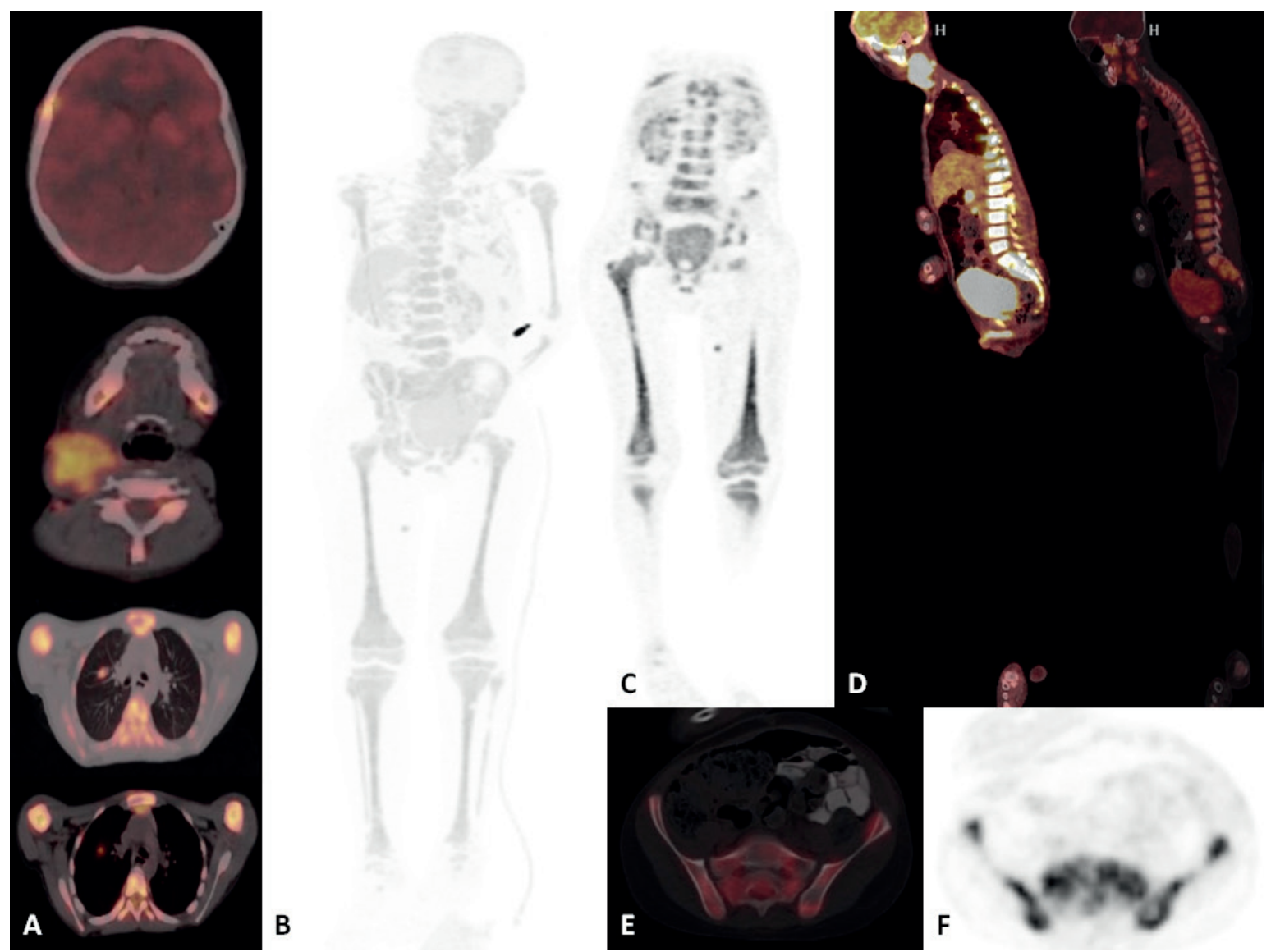

Fig. 2. FDG PET-CT images of a 12 year old male patient with non-Hodgkin's lymphoma diagnosis. Axial fusion (A), Maximum intensity projection (MIP) image (B), coronal PET (C), sagittal fusion (D), axial fusion (E) and axial PET (F) images demonstrate multipl hypermetabolic lymphadenopaties, soft tissue lesions and bone-bone marrow diffuse and multifocal FDG uptake compatible with lymphoma diagnosis. Bone marrow biopsy was positive for this patient.

performed in patients with normal BM activity or increased diffuse activity that suggests reactive bone marrow. ${ }^{7}$ In another pediatric Hodgkin's lymphoma study, PET-CT did not give any false negative result compared to the reference methods $\mathrm{BMB}, \mathrm{CT}$ and magnetic resonance imaging. Furthermore, the specificity and negative predictive value of PET-CT were reported as $100 \%$. Due to the typical multifocal BMI of HL, the authors noted that the sensitivity of $\mathrm{BMB}$ from the iliac crest was low. ${ }^{14}$ For the patients diagnosed with Hodgkin's lymphoma in the current study, the sensitivity and negative predictive value of PET-CT for detecting BMI were both $100 \%$, and its specificity and positive predictive values were $73 \%$ and $44 \%$, respectively. So we can suggest that PET-CT could idendify bone marrow metastatic HL patients as accurately as BMB.

In pediatric patients with a NHL diagnosis, Chen et al. ${ }^{15}$ reported the sensitivity and specificity of PET-CT as $95 \%$ and $98 \%$, respectively based on multifocal accumulations that were not detected by BMB but visualized by PET-CT. In the same study, the sensitivity and specificity of BMB was $56 \%$ and $100 \%$, respectively. However, the authors commented that the role of PET-CT was dependent on the type of lymphoma. ${ }^{15}$ In the current study, for the patients with NHL, the PET-CT sensitivity and specificity were $50 \%$, and its positive and negative predictive values were $60 \%$ and $40 \%$, respectively. These results 

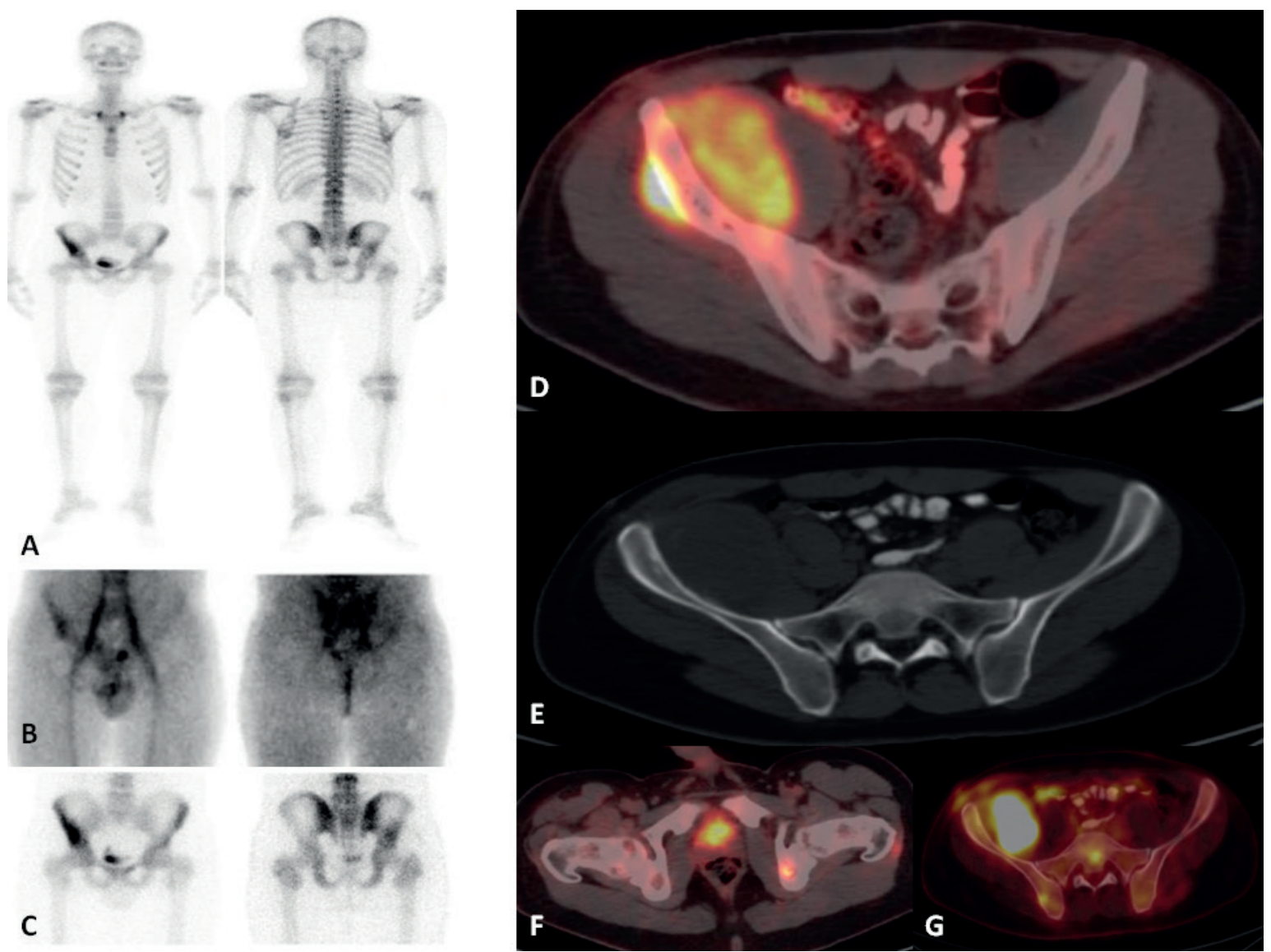

Fig. 3. Images of a 17 year old male patient with Ewing Sarcoma diagnosis. Whole body bone scintigraphy (A), blood pool (B) and statik (C) images demonstrates radioactivity uptake on right iliak crest. FDG PET-CT images of the same patient illustrate both right iliac crest mass and multipl bone-bone marrow FDG uptake on images of axial fusion (D), axial CT (E) and axial fusion pelvic $(F, G)$ images. Bone marrow biopsy was negative for this patient.

support the approach to perform BMB on NHL patients at the time of diagnosis and use PETCT imaging as a complementary method.

In pediatric solid tumors, the number of studies that evaluated BMI using PET-CT is less than those undertaken for lymphomas. In a study conducted with 34 patients with a lymphoma diagnosis accompanied by a solid tumor ( 7 rhabdomyosarcomas, 7 Ewing sarcomas, and 20 neuroblastomas), Zapata et al. $^{6}$ detected no false negative results with PET-CT.

18F-FDG-PET-CT is increasingly used in the diagnosis, staging and prognosis prediction of pediatric neuroblastoma. Li et al. ${ }^{16}$ evaluated 26 patients with a neuroblastoma diagnosis and observed BMI in 16 patients, of whom $10(62.5 \%)$ had bone marrow lesions and 6 $(37.5 \%)$ had diffuse FDG uptake. Taking a positive $\mathrm{BMB}$ as the reference standard, the sensitivity, specificity and accuracy of PET-CT in detecting BMI were found to be 100, 50 and $80.7 \%$, respectively. In addition, PET-CT was able to demonstrate multifocal involvement in 2 patients with a negative result in the iliac crest BMB. ${ }^{16}$ In the current study, the PET-CT specificity and positive predictive value were $100 \%$, and sensitivity and negative predictive value were $75 \%$ and $87.5 \%$, respectively, so we can suggest that PET-CT could idendify non-bone marrow metastatic neuroblastoma patients as accurately as BMB. 
Table III. The distribution of procedures identifying bone marrow involvement according to the primary diagnosis and one-year survival rates.

\begin{tabular}{lcccc}
\hline \multirow{2}{*}{ Diagnosis } & \multicolumn{4}{c}{ BMI status } \\
\cline { 2 - 5 } & PET-CT + / BMB + & PET-CT + / BMB - & PET-CT - / BMB + & PET-CT - / BMB - \\
\hline HL & 4 & 5 & 0 & 14 \\
Survival rate (\%) & 100 & 80 & - & 100 \\
NHL & 6 & 4 & 6 & 4 \\
Survival rate (\%) & 50 & 75 & 50 & 100 \\
Neuroblastoma & 3 & 0 & 1 & 7 \\
Survival rate (\%) & 33 & - & 100 & 100 \\
Ewing sarcoma & 2 & 1 & 2 & 5 \\
Survival rate (\%) & 0 & 0 & 0 & 60 \\
LCH & 1 & 2 & 0 & 3 \\
Survival rate (\%) & 100 & 100 & - & 3 \\
Rhabdomyosarcoma & 2 & 0 & 0 & 33 \\
Survival rate (\%) & 0 & - & - & 00 \\
\hline
\end{tabular}

BMB: bone marrow biopsy, BMI: bone marrow involvement, PET-CT: positron emission tomography-computed tomography, HL: Hodgkin's lymphoma, NHL: non-Hodgkin's lymphoma, LCH: Langerhans cell histiocytosis.

Ewing sarcoma is the second most common malignant bone tumor. In recent years, PETCT has been used for imaging, staging and monitoring treatment response of this tumor. ${ }^{17}$ Kasalak et al. $^{18}$ retrospectively evaluated 38 BMB samples (bilateral in 18 and unilateral in 2 patients) and PET-CT scans belonging to 20 patients with the diagnosis of Ewing sarcoma in terms of BMI. The PET-CT and BMB findings were similar in 36 posterior iliac cristas. According to the patient-based evaluation, the findings from both methods were similar in 19 patients. Considering these results, the authors suggested that in Ewing sarcoma patients, the routine $\mathrm{BMB}$ procedure should be reconsidered when it is possible to perform PET-CT during the evaluation of BMI at the time of diagnosis. ${ }^{18}$ In the current study, in patients with Ewing sarcoma, the PET-CT specificity was $83 \%$, sensitivity was $50 \%$, and positive and negative predictive values were $66.6 \%$ and $71.4 \%$, respectively. In Ewing sarcoma, metastatic status is the most important factor for a risk-adaptive treatment approach. Based on our results, we consider that for accurate staging, BMB should be performed at the time of diagnosis, and PETCT can be used as a supporting method.
The most common soft tissue sarcoma in childhood is rhabdomyosarcoma. With the widespread use of PET-CT imaging, BMB procedure is now frequently employed in the staging of rhabdomyosarcoma. ${ }^{17}$ Federico et al. ${ }^{19}$ reviewed 30 pediatric cases with rhabdomyosarcoma retrospectively and detected $\mathrm{BMI}$ in 4 patients (13\%) by using BMB, of whom 2 also had a positive PET-CT result, and suggested that PET-CT could be used routinely in staging rhabdomyosarcoma. However, in their literature review, Norman et al. ${ }^{20}$ reported that the results of Federico et al. ${ }^{19}$ were not clear, and the sensitivity of PET-CT was limited. In the current study, the specificity, sensitivity, positive and negative predictive values of PETCT for revealing BMI were found to be $100 \%$, so we can suggest that PET-CT could detect BMI as accurately as BMB in rhabdomyosarcoma patients.

$\mathrm{LCH}$ is a reactive proliferative disease of unknown etiology, characterized by the proliferation of langerhans cells. BMI is associated with high risk and low survival, and indicates multisystemic $\mathrm{LCH}$ and poor prognosis. In the literature, the BMI rates were reported as $26.3 \%$ and $33.3 \% .^{21,22}$ In the current study, BMI was presented at a rate of 
$44.4 \%$, and the PET-CT sensitivity, specificity, negative and positive predictive values for demonstrating BMI were $100 \%, 60 \%, 100 \%$, and $33.3 \%$, respectively. So we can suggest that PET-CT could idendify bone marrow metastatic $\mathrm{LCH}$ patients as accurately as BMB. In order not to diagnose multisystemic $\mathrm{LCH}$ based on false positive results, we consider that it is not appropriate to evaluate BMI using the PET-CT method alone.

Limitations of our study are small sample size, the retrospective nature, heterogeneity, only the iliac crest region preference for $\mathrm{BMB}$, and not evaluating the results of other imaging methods. We also know that the involvements we regard as bone marrow metastasis by PET-CT may be paraneoplastic activity. For this reason, the detection of BMI should be investigated by different modalities, and associations between different modality results and survival data should be studied with larger samples prospectively.

In conclusion, the diagnosis should be considered when choosing a procedure to investigate bone marrow involvement in pediatric malignancies. PET-CT may not have high sensitivity and specificity to identify BMI for each type of cancer. The approach of using bilateral BMB and PET-CT as complementary modalities seems reliable.

\section{REFERENCES}

1. Paulino AC, Margolin J, Dreyer Z, Bin S Teh, Stephen Chiang. Impact of PET-CT on involved field radiotherapy design for pediatric Hodgkin lymphoma. Pediatr Blood Cancer 2012; 58: 860-864.

2. Faizan M, Anwar S, Khan S. Demographics and outome in paediatric non-Hodgkin lymphoma: single centre experience at The Children Hospital Lahore, Pakistan. J Coll Physicians Surg Pak 2018; 28: 48-51.

3. Budiongo AN, Ngiyulu RM, Lebwaze BM, et al. Pediatric non-Hodgkin lymphomas: first report from Central Africa. Pediatr Hematol Oncol 2015; 32: 239-249.
4. Khan SJ, Ishaq I, Saeed H, Fayyaz MB, Baqari SAS, Mohammad Wali R. Bone marrow involvement in metastatic pediatric ewing sarcoma. J CollPhysicians Surg Pak 2017; 27: 502-504.

5. Rastogi P, Naseem S, Varma N, et al. Bone marrow involvement in neuroblastoma: a study of hematomorphological features. Indian J Hematol Blood Transfus 2015; 31: 57-60.

6. Zapata $\mathrm{CP}$, Cuglievan B, Zapata CM, et al. PET/CT versus bone marrow biopsy in the initial evaluation of bone marrow infiltration in various pediatric malignancies. Pediatr Blood Cancer 2018; 65: e26814.

7. Hassan A, Siddique M, Bashir $\mathrm{H}$, et al. ${ }^{18} \mathrm{~F}-\mathrm{FDG}$ PETCT imaging versus bone marrow biopsy in pediatric Hodgkin's lymphoma: a quantitative assessment of marrow uptake and novel insights into clinical implications of marrow involvement. Eur J Nucl Med Mol Imaging 2017; 44: 1198-1206.

8. Ozcan Kara P. Pediatrik lenfomalarda PET/BT görüntüleme. Turkiye Klinikleri J Nucl Med-Special Topics 2017; 3: 93-99.

9. Chambers G, Frood R, Patel C, Scarsbrook A. ${ }^{18}$ F-FDG PET-CT in paediatric oncology: established and emerging applications. Br J Radiol 2019; 91: 20180584 .

10. Tatsumi M, Miller JH, Wahl RL. 18F-FDG PET/CT in evaluating non-CNS pediatric malignancies. J Nucl Med 2007; 48: 1923-1931.

11. Odalovi'c S, Sobic-Saranovic D, Pavlovic S, et al. Preliminary experience with 18f-fluoro-deoxyglucose positron emission tomography/computed tomography in pediatric oncology patients. Acta Chir lugosl 2011; 58: 67-73.

12. Wegner EA, Barrington SF, Kingston JE, et al. The impact of PET scanning on management of paediatric oncology patients. Eur J Nucl Med Mol Imaging 2005; 32: 23-30.

13. Cheng G, Chen W, Chamroonrat W, Drew A Torigian, Hongming Zhuang, Abass Alavi.Biopsy versus FDG PET/CT in the initial evaluation of bone marrow involvement in pediatric lymphoma patients. Eur J Nucl Med Mol Imaging 2011; 38: 1469-1476.

14. Purz S, Mauz-Korholz C, Korholz D, et al. $\left[{ }^{18} \mathrm{~F}\right]$ fluorodeoxyglucose positron emission tomography for detection of bone marrow involvement in children and adolescents with Hodgkin's lymphoma. J Clin Oncol 2011; 29: 3523-3528.

15. Chen S, Wang S, He K, Ma C, Fu H , Wang H. PET/ $\mathrm{CT}$ predicts bone marrow involvement in paediatric non-Hodgkin lymphoma and may preclude the need for bone marrow biopsy in selected patients. Eur Radiol 2018; 28: 2942-2950. 
16. Li C, Zhang J, Chen S, et al. Prognostic value of metabolic indices and bone marrow uptake pattern on preoperative 18F-FDG PET/CT in pediatric patients with neuroblastoma. Eur J Nucl Med Mol Imaging 2018; 45: 306-315.

17. Harrison DJ, Parisi MT, Shulkin BL. The role of ${ }^{18} \mathrm{~F}-\mathrm{FDG}-\mathrm{PET} / \mathrm{CT}$ in pediatric sarcoma. Semin Nucl Med 2017; 47: 229-241.

18. Kasalak O, Glaudemans AWJM, Overbosch J, Jutte PC, Kwee TC . Can FDG-PET/CTreplace blind bone marrow biopsy of the posterior iliac crest in Ewing sarcoma? Skeletal Radiol 2018; 47: 363-367.

19. Federico SM, Spunt SL, Krasin MJ, et al. Comparison of PET-CT and conventional imaging in staging pediatric rhabdomyosarcoma. Pediatr Blood Cancer 2013; 60: 1128-1134.
20. Norman G, Fayter D, Lewis-Light K, et al. An emerging evidence base for PET-CT in the management of childhood rhabdomyosarcoma: systematic review. BMJ Open 2015; 5: e006030.

21. Kumar M, Singh Sachdeva MU, Naseem S, et al. Bone marrow infiltration in Langerhan's cell histiocytosis - An unusual but important determinant for staging and treatment. Int J Hematol Oncol Stem Cell Res 2015; 9: 193-197.

22. Goyal A, Rani S, Singh T, Choudhury P, Dubey A. Childhood histiocytoses: a review of twenty two cases. Indian Pediatr 1998; 35: 151-156. 